\title{
A Sensor Data-Based Approach for the Definition of Condition Taxonomies for a Hydraulic Pump ${ }^{\dagger}$
}

\author{
Carlos Gil Buiges ${ }^{1,2}$ and Caroline König ${ }^{1,2, *}$ \\ 1 Department of Computer Science, Universitat Politècnica de Catalunya, UPC BarcelonaTech, \\ 08034 Barcelona, Spain \\ 2 ISA DATA S.L, San Joan Despi, 08970 Barcelona, Spain \\ * Correspondence: ckonig@cs.upc.edu \\ $+\quad$ Presented at the 7th Electronic Conference on Sensors and Applications, 15-30 November 2020; \\ Available online: https:/ /ecsa-7.sciforum.net/.
}

Published: 15 November 2020

check for

\begin{abstract}
Condition monitoring $(\mathrm{CM})$ is an important application in industry for detecting machine failures in an incipient stage. Based on sensor data, computational intelligence methods provide efficient solutions for the analysis of high dimensional process data with the ability to detect and predict complex condition states. IOT gateways are affordable devices with the allow to implement data ingestion and data analytics task on an edge device providing the possibility to implement condition monitoring in real-time on the device. In this work, we present an experimental bench for the sensorization of a hydraulic installation based on IoT gateways in order to detect several blocking states in a hydraulic pump and to avoid the cavitation problem. The experiments of 15 different blocking conditions yield a novel dataset with process sensor information for the described problem. The dataset is analyzed from a data quality point of view to find a meaningful categorization of fault conditions, which are feasible to be implemented in a condition monitoring system. We use an exploratory data analysis approach, which is based on principal component analysis provides data visualization of the different blocking conditions of the experiment, and allows us to decide on a proper fault categorization by detecting clearly separated data groups.
\end{abstract}

Keywords: condition monitoring; hydraulic pump; IoT gateway; node-red; fault taxonomies

\section{Introduction}

Nowadays, the industry is facing a transition to a new paradigm where the collection and process of data are taking a significant role in the improvement of technology. An important application of computational intelligence methods in industry is condition monitoring. Those methods analyze sensor data, which reflect the physical condition of the machine and predict the state of a subsystem [1] or failure conditions in an early stage. The integration of computational intelligence applications with machines postulate several technical challenges. First the need to develop machine learning models for effective and secure detection from process and sensor data and second its integration with the device for real-time detection [2]. Machine learning is a data-driven approach, therefore being the data quality paramount. From a machine learning point of view, the design of the condition monitoring system should be studied carefully in order to decide on relevant sensor information and a proper categorization of fault conditions for which the machine learning model will be trained. In order to build a reliable condition monitoring system it is important to recollect information from representative states of the working condition of the machine. This is not always possible, because machine manufacturers are not always willing to run their machines until failure, therefore being data of 
normal working conditions much more abundant than data for failure conditions and degradation states [3].

The present research focuses on the detection of blockages in hydraulic circuits. The main reason why blockages have an important impact on the health of a hydraulic system stands on its non-desired effects on pumps. With a little research about the main reasons for the substitution of rotors in pumps, we can find cavitation as one of the most exhausting phenomena produced inside pumps. Moreover, it also can lead to other defects as bearing and rotor faults, which are related, among others, with undesired vibrations and thermal and dynamic stress [4]. The relation between flow blockages and cavitation resides in the pressure drop produced when the section of a pipe is reduced [5]. This is because cavitation is produced when the pressure and temperature of a fluid reach the boiling point during a short period of time. Then, there are three ways of producing cavitation: raising the temperature of a fluid, decreasing its pressure, or combining both. The physical principles that govern cavitation are that when the pressure of the fluid is low enough, it evaporates locally producing a bubble that travels through the pump until it reaches a higher-pressure point. Then, the gas is turned back to liquid in such a way that the bubble implodes, generating a shockwave that exhausts the closest obstacles. Long exposure to cavitation can produce severe damage in the inner part of the pumps. To achieve a reliable blockage detection, there are two challenges to face : (1) the implementation of sensors that allow us to know the state of the circuit, (2) and the ingestion and analysis of the data in real-time and as close as possible to the installation. This last concept is often referred to as edge computing, which is, processing the data on-site [6].

In this study, a closed-loop hydraulic system has been built using different types of process sensors to capture information about the different performing working states. These states regard to blockages artificially induced in the inlet and outlet pipes of the pump. The collected process data was analyzed from a data analytical point of view to find substantial differences between a non-blockage and a blockage state. The objective is to design a condition monitoring for the pump, therefore an exploratory data analysis on the sensor data is carried out to assess data quality.

Ultimately, this document limits to explain the test bench installation, the sensor data integration approach, signal transformations and the exploratory data analysis. As a result, a sensor data set for the described experiments is released. The machine learning model for condition monitoring will be tackled in ongoing research. The remaining part of the article is structured as follows. A material section (Section 2) with a description of the hydraulic circuit, the data ingestion system from sensors and the description of the conducted experiments. Section 3 explains the exploratory data analysis on the fault categories followed by the conclusions of the research.

\section{Materials}

\subsection{Experimental Bench}

The experimental bench of the hydraulic system is composed by a pump, a water tank, sensors, actuators and the pipes (Figure 1). It has been implemented in a closed-loop topology. In this circuit, we can differentiate two parts, the inlet side, and the outlet side. These parts are defined by the position of the pump. In the inlet side of the circuit, there is the flow rate sensor (F1). Next to it, there is the inlet valve (FCV1), which has been placed to simulate flow blockages. For the outlet side, there is the pressure sensor (P2) and the outlet valve (FCV1) with the same goal as the inlet valve. Beside pressure and flow control, there are three more signals monitored: firstly, the vibrations of the pump cage (ACC); secondly, the temperature of the pump cage (NTC); and thirdly, the current consumption of the pump (PC). The temperature transducer has been placed to control the condition of the pump during the tests to prevent possible overheating. Despite this, the temperature has not been considered in the data set because its behaviour doesn't depend on the current test, but also on the previous ones and on the amount of time that the pump has been turned on. The hydraulic pump is a self-priming centrifugal pump (Model Einhell GC-GP6538). 

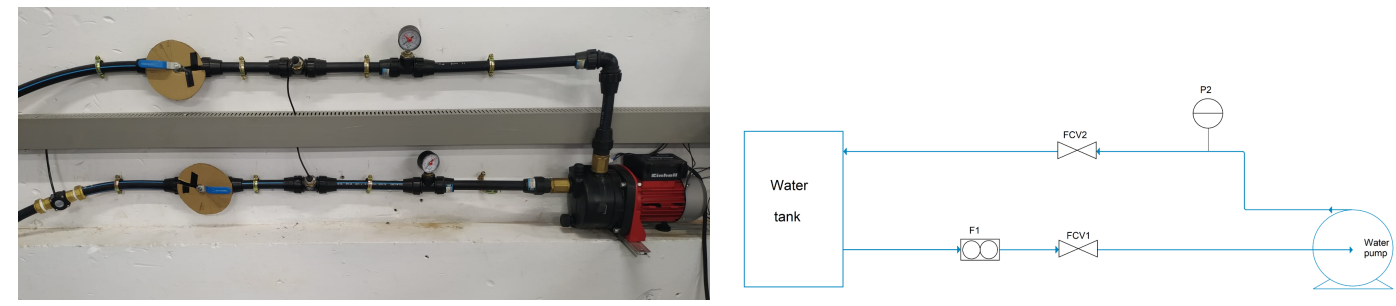

Figure 1. Image and schematic representation of the test bench.

Regarding the sensors, Table 1 shows details about the sensor model, the measured magnitudes and the abbreviation (sensor tag) used in this document.

Table 1. Description of sensors.

\begin{tabular}{ccccc}
\hline Sensor Tag & Model & Range & Measured Variable & Unit \\
\hline P2 & Walfront 1PC G3/8 0-2 BAR & $0-2$ bar & Outlet pressure & $\mathrm{bar}$ \\
F1 & FS300A & $1-60 \mathrm{~L} / \mathrm{Min}$ & Flow rate & $\mathrm{L} / \mathrm{s}$ \\
A1 & ADXL335 & L/s & One axe vibration & $\%$ \\
NTC1 & MF52B NTC & $200^{\circ} \mathrm{C}$ & Pump cage temperature & ${ }^{\circ} \mathrm{C}$ \\
PC1 & SZT 15-CH-10 & $0-10 \mathrm{~A}$ & Pump power consumption & $\mathrm{A}$ \\
\hline
\end{tabular}

\subsection{Data Ingestion}

The process sensor data was registered in an IoT 4.0 system, which includes a PLC S7 1200 and an IoT2040 gateway, both Siemens devices. The PLC is responsible for translating the analogic signals from the sensors to digital information and communicate it via the modbus protocol to the IOT gateway. On the other hand, the IOT2040 gateway processes and stores the data. In this study, the IOT2040 gateway was configured for data ingestion and signal transformation via the Node-RED utility. The sampling frequency of the data was configured according to the variability of the measurements using two different sampling frequencies: for pressure, flow rate and temperature, the frequency of sampling has been $4 \mathrm{~Hz}$; for the current consumption and vibration, the sampling rate has been $20 \mathrm{~Hz}$. In the first group, the frequency is lower since these three magnitudes are more stable. Instead, current consumption and vibration are signals with more relevant harmonics that can reflect patterns related to the behaviour of the pump.

The Node-RED implementation is responsible for (1) data collection and transformation, (2) data storage and (3) data visualization. The first process (data collection and transformation) comprises the collection of the data coming from the PLC through a preset modbus Node-RED block and the calculation of the transformed signals - mean, standard deviation and the root mean square (RMS) in real-time. (Figure 2).

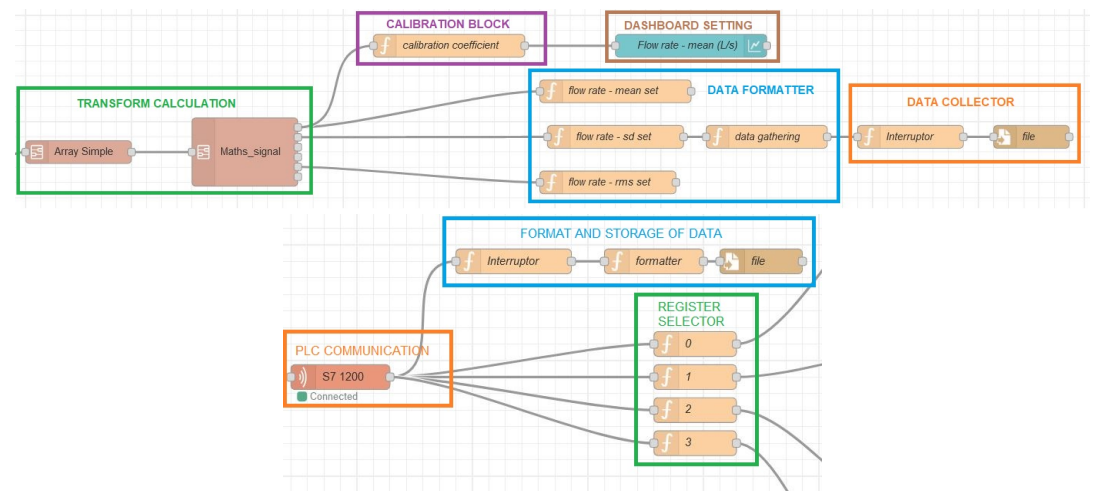

Figure 2. Node-RED data integration. 


\subsection{Experiments}

There have been conducted 16 experiments on the test bench to take measurements under different levels of blockage. The experiments consist of setting three different blockage levels (20\%, $50 \%$ and $80 \%)$ for both valves in the circuit and test different combinations of blockage of the inlet and outlet valve. Table 2 shows the corresponding blockage conditions of the inlet and outlet valve for each experiment. Experiments NO1 and NO2 correspond to normal working conditions of the circuit without blockage. Experiments IV1-IV3 correspond to blocking solely at the inlet valve, while experiments OV1-OV3 represent different degree of blocking in the outlet valve. Experiments IO correspond to simultaneous blockage at the inlet and outlet valve with different levels of blockage ranging from $20 \%$ to $80 \%$.

Table 2. Tested scenarios.

\begin{tabular}{lccccc}
\hline & & \multicolumn{4}{c}{ Outlet Valve } \\
& & $\mathbf{0 \%}$ & $\mathbf{2 0} \%$ & $\mathbf{5 0} \%$ & $\mathbf{8 0} \%$ \\
\hline \multirow{3}{*}{ Inlet valve } & $\mathbf{0 \%}$ & NO1/NO2 & OV1 & OV2 & OV3 \\
& $\mathbf{2 0 \%}$ & IV1 & IO11 & IO12 & IO13 \\
& $\mathbf{5 0 \%}$ & IV2 & IO21 & IO22 & IO23 \\
& $\mathbf{8 0 \%}$ & IV3 & IO31 & IO32 & IO33 \\
\hline
\end{tabular}

Except for the normal condition experiments, every experiment was conducted in the following three phases: (1) normal operation for $1 \mathrm{~min}$, (2) operation with blockage for $3 \mathrm{~min}$ and (3) normal operation again for $1 \mathrm{~min}$. Figure 3 shows the measurements of the 4 sensors (acceleration, flow rate, power consumption and pressure) throughout the different phases of the experiments. For each experiment the mean, root mean square (RMS) and standard deviation is shown for the respective sensor signals after standardization. The different experimental phases can be identified in the registered data as the moment of transition between normal and abnormal (or blockage) state is known.

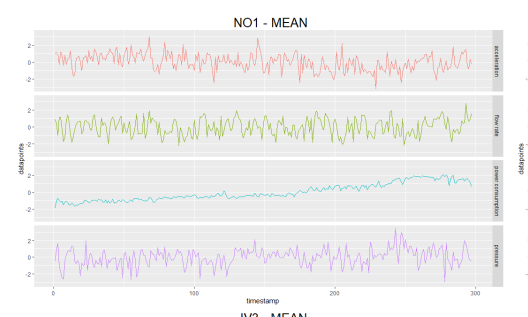

IV3-MEAN

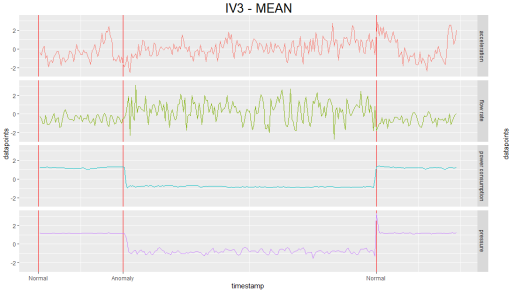

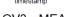

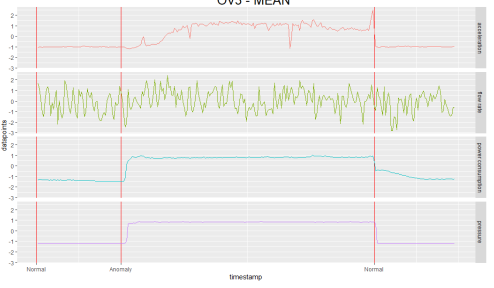

NO1-RMS

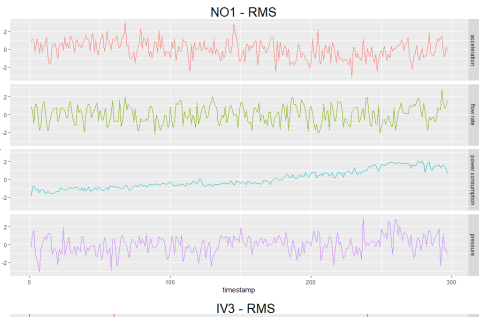

IV3-RMS

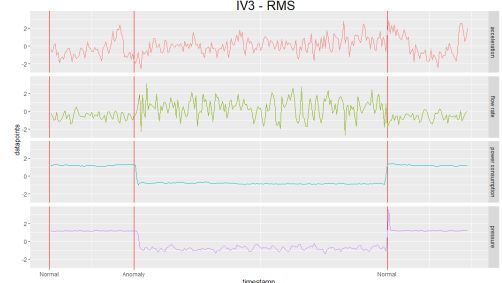

$\underset{\text { OV3-RMS }}{\stackrel{3}{30}}$

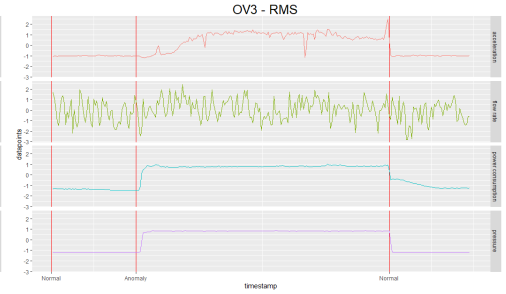

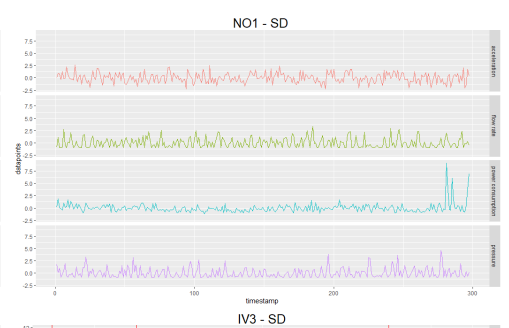
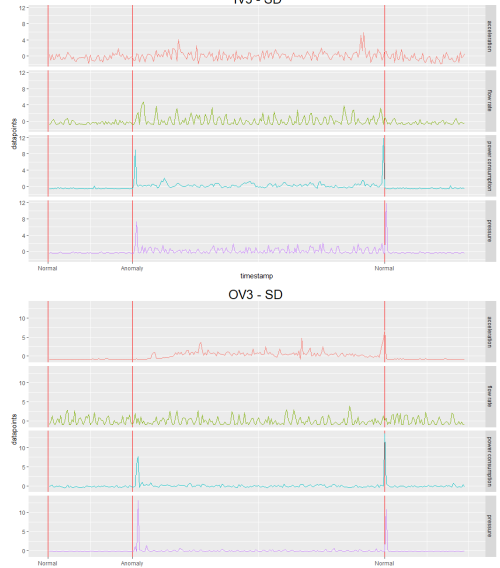

Figure 3. Sensor signals for normal state (NO1), inlet valve blockage (IV3), outlet valve blockage (OV3) experiments. Mean, RMS and standard deviation metrics are shown from left to right.

A visual comparison of the measurement of the different experiments reveals some of the effects of blockage. With inlet valve blockage (IV3 experiment) the power consumption of the pump and 
the pressure drops during the abnormal state. Blockage in the outlet valve (OV3 experiment) has the opposite effect and increases the power consumption and pressure. Non-obvious relationships between sensor measurements should be analyzed with statistical methods.

\section{Exploratory Data Analysis}

This section presents the results of an exploratory data analysis of the sensor data to gain further insights about the data quality and separability among the different blockage states of the pump. Exploratory data analysis finds a wide application as a first investigation on data to reveal patterns and derive useful knowledge about the data under study. Statistical validations and graphical visualizations are common tools for data exploration [7]. The aim of the conducted experiments is the construction of a condition monitoring system for the pump and the recognition different blockage or failure states. Therefore, it is important to evaluate in an initial phase whether the information captured from sensors establish representative categories of the machine's state.

For this analysis we visualize the sensor data in a lower dimensional space. The original data set comprises 12 attributes - three transformations (mean, RMS and standard deviation) for each of the 4 sensor signals. In the following, the results of dimensionality reduction with Principal Component Analysis (PCA) on the data set is shown. This mathematical method generates new reference axes (principal components) in the directions with greatest variance [8].The objective of PCA, then, is to diminish the number of dimensions with the smallest loss of information possible.

Figure 4 shows the visualization of the sensor data set after PCA transformation in three dimensions. Apparently, the PCA visualization of all 16 conditions does not show a clear separation of states. In consequence, we decide to compare data separability between selected states of the system, i.e. the normal state and different levels of blockage. We establish the categories of soft blockage, medium blockage and hard blockage considering a level of $20 \%, 50 \%$ and $80 \%$ of blockage in the valves in each category. We also consider the case of progressive degree of blockage in both valves in the diagonal category. The category labeled as extreme considers blockage at $80 \%$ in either the inlet or the outlet valve (IV3 or OV3) or both of them (IO33). Table 3 gives an overview of the blockage states involved in each category.

Table 3. Taxonomies of condition states.

\begin{tabular}{|c|c|c|c|c|c|c|c|c|c|c|}
\hline Level & Id & & States & & Level & Id & & States & & \\
\hline \multirow{2}{*}{ Soft block } & S1 & NO1 & IO11 & & & H1 & NO1 & $\mathrm{IO} 33$ & & \\
\hline & S2 & NO1 & IV1 & OV1 & Hard block & $\mathrm{H} 2$ & NO1 & OV3 & IV3 & \\
\hline \multirow{2}{*}{ Medium block } & M1 & NO1 & $\mathrm{IO} 22$ & & Diagonal & D & NO1 & IO11 & $\mathrm{IO} 22$ & IO33 \\
\hline & M2 & NO1 & IV2 & OV2 & Extremes & E & NO1 & IO33 & IV3 & OV3 \\
\hline
\end{tabular}

Figure 4 shows the PCA visualization of each of the blockage categories. The states of the soft blockage category show some overlapping and are less clearly separated from the normal state than the medium or hard blockage states. With increasing level of blockage the groups appear more clearly separated in the PCA plot. Although medium blockage category already reveals differences, this separation is clear with the hard blockage and extreme blockage states, both categories representing differences of $80 \%$ of blockage. Interestingly, the progressive blockage in both valves (represented in the diagonal group) reveals overlapping between states. We interpret that the states of simultaneous blockage in both valves may be more difficult to detect due to underlying similarities in the sensor data. 

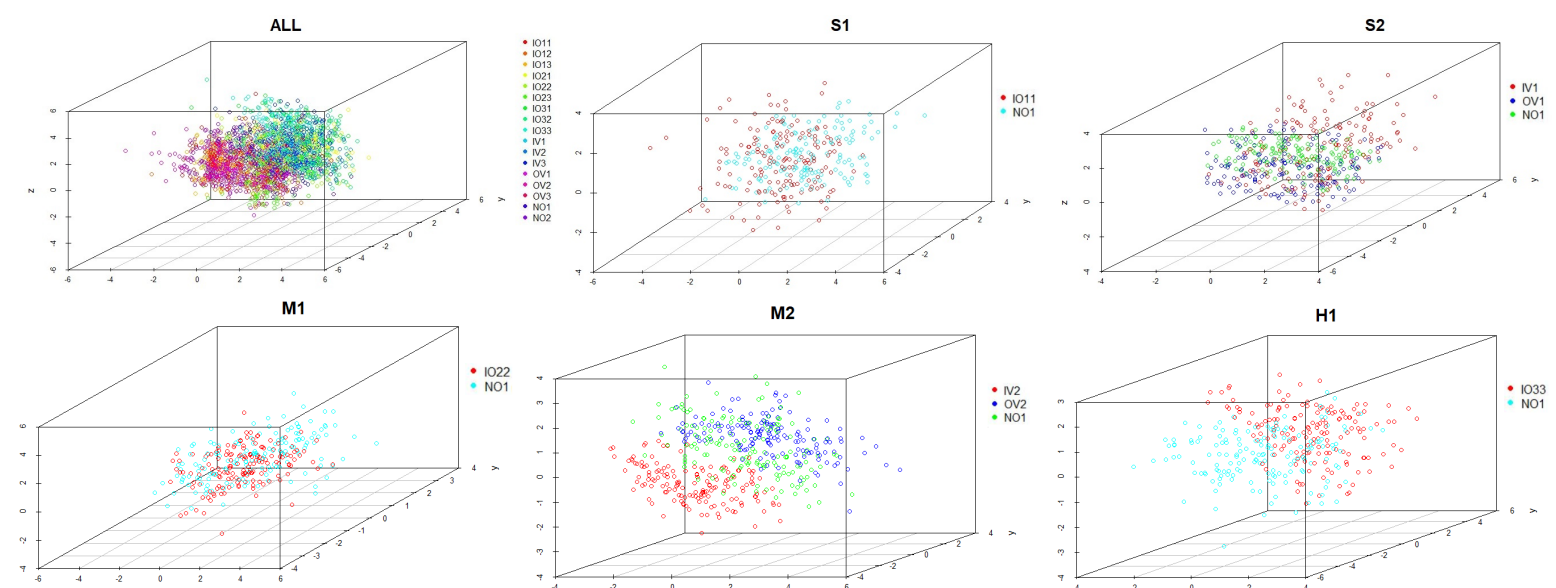

M2
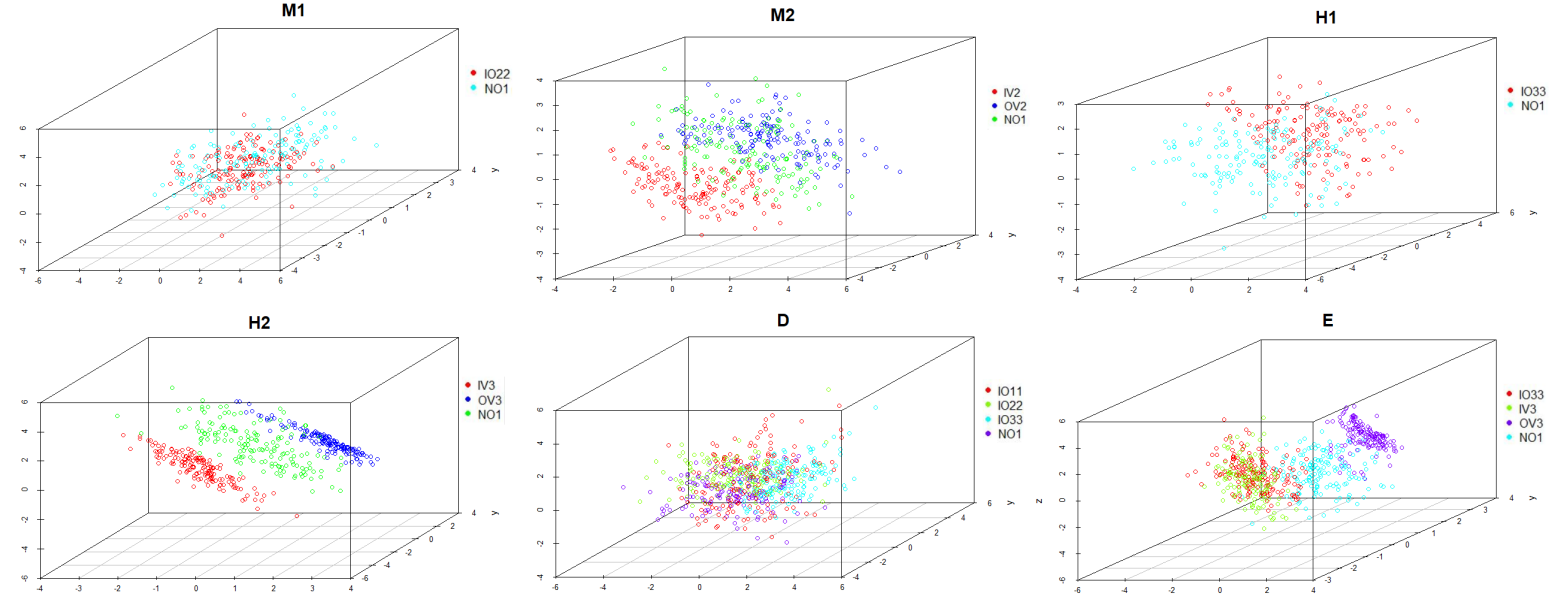

Figure 4. PCA visualization of the state groupings.

\section{Conclusions}

The experiments on the test bench provided a new data set of sensor information for the investigation of different blockage states in hydraulic circuits. The exploratory analysis of the data set identified the blocking states with a substantial differences to the non-blockage state. This study is a preliminary step in the construction of a condition monitoring system for pumps. The design of the machine learning system will be carried out in ongoing research and relies on the identification of relevant blockage states carried out in this study.

Author Contributions: conceptualization, C.K. and C.G.B.; methodology, C.K.; software, C.G.B.; validation,formal analysis and investigation: C.G.B. and C.K.; resources and data curation: C.G.B.; writing-review and editing, C.K. and C.G.B.; visualization, C.G.B. and C.K; supervision, C.K. All authors have read and agreed to the published version of the manuscript.

Conflicts of Interest: The authors declare no conflict of interest.

\section{References}

1. Higgs, P.A.; Parkin, R.; Jackson, M.; Al-Habaibeh, A.; Zorriassatine, F.; Coy, J. A survey on condition monitoring systems in industry. In Proceedings of the ASME 7th Biennial Conference on Engineering Systems Design and Analysis, Manchester, UK, 19-22 July 2004; pp. 163-178.

2. Murshed, M.G.; Murphy, C.; Hou, D.; Khan, N.; Ananthanarayanan, G.; Hussain, F. Machine learning at the network edge: A survey arXiv 2019, arXiv:1908.00080.

3. Qiu, H.; Lee, J.; Lin, J.; Yu, G. Robust performance degradation assessment methods for enhanced rolling element bearing prognostics. Adv. Eng. Informatics 2003, 17, 127-140.

4. Kumar, S.; Mukherjee, D.; Guchhait, P.K.; Banerjee, R.; Srivastava, A.K.; Vishwakarma, D.; Saket, R. A comprehensive review of condition based prognostic maintenance (CBPM) for induction motor. IEEE Access 2019, 7, 90690-90704.

5. Franc, J.; Michel, J. Fundamentals of Cavitation; Springer Science \& Business Media: Berlin/Heidelberg, Germany, 2006; Voume 76.

6. Yousefpour, A.; Fung, C.; Nguyen, T.; Kadiyala, K.; Jalali, F.; Niakanlahiji, A. All one needs to know about fog computing and related J. Syst. Archit. 2019, 98, 289-330. 
7. Jebb, A.T.; Parrigon, S.; Woo, S.E. Exploratory data analysis as a foundation of inductive research. Hum. Resour. Manag. Rev. 2017, 27, 256-276.

8. Pearson, K. On lines and planes of closest fit to systems of points in space. Lond. Edinb. Dublin Philos. Mag. J. Sci. 1901, 2, 559-572.

Publisher's Note: MDPI stays neutral with regard to jurisdictional claims in published maps and institutional affiliations.

(C) 2020 by the authors. Licensee MDPI, Basel, Switzerland. This article is an open access article distributed under the terms and conditions of the Creative Commons Attribution (CC BY) license (http://creativecommons.org/licenses/by/4.0/). 